\title{
Epidemiología y resultado estético funcional de la reducción de la fractura nasal
}

\section{Epidemiology and aesthetic and functional outcome in nasal bone fracture reduction}

Gustavo Bravo C1', Daniel Muñoz S², Christian Olavarría L'1, Carla Pereira P².

\section{RESUMEN}

Introducción: Las lesiones faciales traumáticas más frecuentes son las fracturas nasales debido a la prominencia de la pirámide nasal. Existe cierto consenso en que cuanto antes se intente su reducción más posibilidades existen de obtener un resultado exitoso.

Objetivo: Describir epidemiológicamente y evaluar los resultados funcionales y estéticos de las fracturas.

Material y método: Se revisaron las fichas clínicas de los pacientes para describir los datos epidemiológicos, se realizó la encuesta SNOT-22 para evaluar los resultados funcionales y una encuesta cualitativa para la percepción estética de las reducciones.

Resultados: De un total de 42 pacientes, predominó el sexo masculino 6:1, la edad promedio fue de 34,4 años. El 83,3\% de la reducciones se realizó en policlínico con anestesia local y el procedimiento se realizó en promedio 8,6 días posfractura. Sobre el resultado funcional destaca la obstrucción nasal posreducción en 43,6\% con un puntaje SNOT-22 promedio de 46,9 puntos y el 47,62\% consideró su resultado estético como peor.

Discusión: Consideramos el resultado estético funcional como no satisfactorio, por lo que no sería inadecuado realizar cirugía de rinoseptoplastía inmediata o diferida para mejorar el resultado.

Palabras clave: Fractura nasal, calidad de vida, resultado estético.

\section{ABSTRACT}

Introduction: Nasal fractures are the most common facial injuries because of the prominence of the nose. There is some consensus that the sooner you try their reduction more likely is a successful outcome.

Aim: Epidemiologically describe and evaluate functional and aesthetic results of nasal fractures.

\footnotetext{
1 Médico, Servicio de Otorrinolaringología Hospital Clínico Universidad de Chile.

2 Médico. Universidad de Chile.
} 
Material and method: We reviewed the medical records of patients to describe the epidemiological data, perform the SNOT-22 survey to evaluate the functional results and a qualitative survey for aesthetic perception of the reductions.

Results: Of a total of 42 patients, males predominated 6:1, average age was 34.4 years. $83.3 \%$ of the reductions took place in clinic with local anesthesia and the procedure was performed on average 8.6 days post-fracture. With regard to functional outcome highlights only nasal obstruction after reduction of $43.6 \%$ with a SNOT-22 scoring average of 46.9 points and $47.62 \%$ of patients considered a worse cosmetic outcome.

Discussion: We believe that the functional and aesthetic result is not satisfactory, so we would recommend a septorhinoplasty immediate or deferred for better results.

Key words: Nasal bone fracture, quality of life, aesthetic outcome.

\section{INTRODUCCIÓN}

La nariz es susceptible de heridas traumáticas debido a su posición central en la cara y la proyección anterior de la pirámide nasal. La fractura nasal corresponde a la fractura facial más frecuente y a la tercera fractura más común del esqueleto humano ${ }^{1}$. Las causas más frecuentes de esta fractura son agresiones físicas, caídas accidentales, accidentes deportivos y de tránsito. Su diagnóstico es eminentemente clínico, con confirmación radiológica ya sea con radiografía de huesos propios nasales o tomografía computarizada².

El tratamiento consiste en hacer una reducción de la fractura, convencionalmente entre 7 y 10 días posterior a la fractura, sin embargo, existe cierta noción en que cuanto antes se intente su reducción más posibilidades existen de obtener un resultado exitos $0^{3}$, utilizando para ello anestésicos locales y en algunos casos con anestesia general, dependiendo del grado de cooperación del paciente. La reducción cerrada se ha asociado con 10\%-50\% de deformidad nasal posreducción, por lo cual necesitaría de rinoplastía o septoplatía posterior ${ }^{1}$.

La necesidad actual de optimizar la satisfacción del paciente, sobre todo con lo relacionado a estructuras faciales, nos hace cada vez más revisar nuestras técnicas e innovar en busca de mejores resultados.

\section{OBJETIVO}

Nuestro objetivo es describir los aspectos epidemiológicos y evaluar los resultados funcionales y estéticos de las fracturas nasales reducidas en el Servicio de Otorrinolaringología del Hospital Clínico de la Universidad de Chile.

\section{MATERIAL Y MÉTODO}

Estudio descriptivo transversal. Se revisaron las fichas clínicas de los pacientes con fractura nasal tratados en el Servicio de Otorrinolaringología del Hospital Clínico de la Universidad de Chile entre julio y diciembre de 2009. Se extrajeron datos epidemiológicos, del proceso diagnóstico y tratamiento. Además, se realizó la encuesta SNOT-22 modificada (Tabla 1) para evaluar los resultados funcionales de la reducción durante entrevista y examen clínico al cual se citó a los pacientes, principalmente en lo relacionado a la presencia de obstrucción nasal y aparición de roncopatía.

Para evaluar la percepción del resultado estético de la reducción, durante la entrevista se preguntó por este hecho, calificándolo de "mucho mejor", "mejor", "igual", "peor" o "mucho peor" que antes de la fractura nasal.

Para el análisis estadístico se utilizó el software Stata 10.1 (StataCorp. 2008. College Station, TX: StataCorp LP.) expresando en mediana y desviación estándar las variables continuas y en proporciones (porcentajes) las variables categóricas. Para estas últimas se utilizaron las pruebas de Chi cuadrado y prueba exacta de Fisher. Para correlacionar la relación entre percepción estética y funcional usamos la prueba de Kruskal Wallis para comparación entre varios grupos. Se consideró un valor $p$ significativo menor a 0,05 . 
Tabla 1. Sino-Nasal Outcome Test modificado (SNOT-22)

\begin{tabular}{|c|c|c|c|c|c|c|c|}
\hline 1. & Necesidad de limpiar la nariz & 0 & 1 & 2 & 3 & 4 & 5 \\
\hline 2. & Picazón nasal & 0 & 1 & 2 & 3 & 4 & 5 \\
\hline 3. & Rinorrea & 0 & 1 & 2 & 3 & 4 & 5 \\
\hline 4. & Obstrucción nasal & 0 & 1 & 2 & 3 & 4 & 5 \\
\hline 5. & Pérdida olfato o sabor & 0 & 1 & 2 & 3 & 4 & 5 \\
\hline 6. & Tos & 0 & 1 & 2 & 3 & 4 & 5 \\
\hline 7. & Descarga nasal posterior & 0 & 1 & 2 & 3 & 4 & 5 \\
\hline 8. & Descarga nasal filante & 0 & 1 & 2 & 3 & 4 & 5 \\
\hline 9. & Sensación oído tapado & 0 & 1 & 2 & 3 & 4 & 5 \\
\hline 10. & Mareos & 0 & 1 & 2 & 3 & 4 & 5 \\
\hline 11. & Dolor de oídos & 0 & 1 & 2 & 3 & 4 & 5 \\
\hline 12. & Dolor facial & 0 & 1 & 2 & 3 & 4 & 5 \\
\hline 13. & Insomnio & 0 & 1 & 2 & 3 & 4 & 5 \\
\hline 14. & Despierta de noche & 0 & 1 & 2 & 3 & 4 & 5 \\
\hline 15. & Sueño superficial & 0 & 1 & 2 & 3 & 4 & 5 \\
\hline 16. & Despierta cansado & 0 & 1 & 2 & 3 & 4 & 5 \\
\hline 17. & Fatiga & 0 & 1 & 2 & 3 & 4 & 5 \\
\hline 18. & Baja productividad & 0 & 1 & 2 & 3 & 4 & 5 \\
\hline 19. & Baja concentración & 0 & 1 & 2 & 3 & 4 & 5 \\
\hline 20. & Frustración/irritabilidad & 0 & 1 & 2 & 3 & 4 & 5 \\
\hline 21. & Tristeza & 0 & 1 & 2 & 3 & 4 & 5 \\
\hline 22. & Avergonzado & 0 & 1 & 2 & 3 & 4 & 5 \\
\hline
\end{tabular}

\section{RESULTADOS}

\section{Aspectos clínicos y epidemiológicos}

De un total de 42 pacientes, predominó el sexo masculino $(83,3 \%)$ con una edad de $34,4 \pm 16,4$ años. Todos los pacientes fueron evaluados con radiografía de huesos propios nasales y en 6 de ellos $(14,2 \%)$ se requirió de tomografía computarizada para precisar el diagnóstico y su extensión.

Con respecto al mecanismo de trauma nasal encontramos que en proporción mayoritaria $(76,17 \%)$ las fracturas se debieron a accidentes deportivos 0 agresiones físicas, seguidas de más atrás por accidentes vehiculares y caídas accidentales. El desglose de los mecanismos se presenta en la Figura 1.

El procedimiento de reducción se realizó de manera ambulatoria con anestésicos locales en el $83,3 \%$ de los pacientes. El resto (7 pacientes) requirió de sedación anestésica general con intubación orotraqueal, dependiendo del grado de cooperación en el caso de los niños y en adultos con fracturas complejas (2 pacientes, que requirieron de reducción abierta de su fractura nasal). En términos generales el procedimiento de reducción de la fractura nasal fue realizado $8,6 \pm 5,4$ días

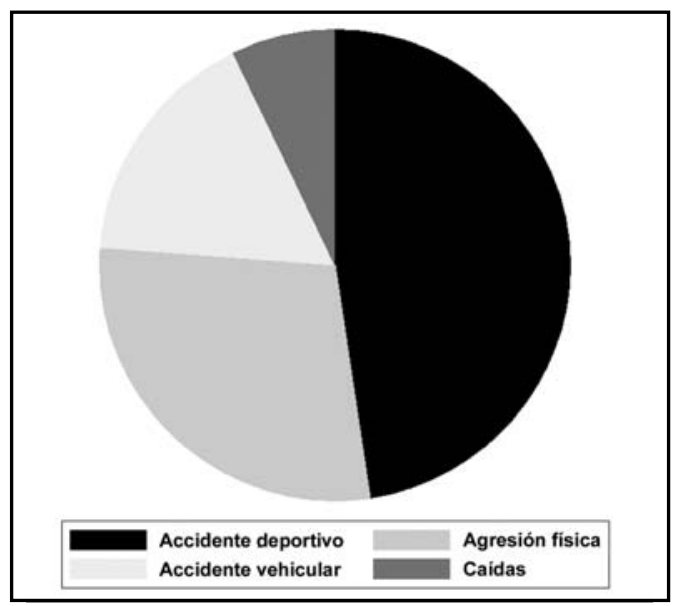

Figura 1. Distribución de mecanismos de trauma en pacientes con fractura nasal, tratados en el Hospital Clínico de la Universidad de Chile. 
posteriores al daño inicial, sin diferencia significativa por $\operatorname{sex} 0(p=0,2)$.

\section{Resultado estético y funcional}

Sobre el resultado funcional destaca la presencia de obstrucción nasal, de acuerdo a las respuestas del SNOT-22, en el 43,6\% de los casos. En estos pacientes el examen clínico evidencia mayor desplazamiento de huesos propios y desviación septal. El puntaje SNOT-22 fue en general de $46,97 \pm 10,4$, en un rango de 27 a 71 puntos, sin diferencia significativas por sexo $(p=0,09)$.

El $73,81 \%$ de los pacientes presentó roncopatía, al extraer las respuestas de esta índole de la encuesta, con predominio del sexo masculino $(83,8 \%)$. Cabe señalar que en este grupo de pacientes, se tiene el antecedente de roncopatía previa al traumatismo en el $73,3 \%$ de ellos (22 de los 31 pacientes que la presentaron posterior a la reducción).

Con respecto a la autopercepción estética de las reducciones de las fracturas nasales, los pacientes consideraron su resultado como "mejor que antes" en el $11,9 \%(n=5)$, "igual que antes" el 40,48\% ( $n=17)$ y "peor que antes" el
$47,62 \%(n=20)$. Las otras alternativas no aparecieron como respuesta en nuestra serie de pacientes.

Al correlacionar la percepción estética con el resultado funcional (puntaje SNOT-22) encontramos que aquellos pacientes que se consideraron mejor que antes obtuvieron un puntaje considerablemente menor que aquellos que se consideraron "peor que antes" (32,8 puntos versus 51,3, respectivamente). Esta diferencia fue estadísticamente significativa $(p<0,0001)$ (Figura 2).

\section{DISCUSIÓN}

El Sino-Nasal Outcome Test (SNOT) fue originalmente desarrollado como una escala de evaluación de salud en pacientes con rinosinusitis. Sin embargo, hace algunos años ha aparecido su uso en la evaluación de patología nasal, principalmente con la incorporación de 2 ítemes extras que aluden a la presencia o no de obstrucción nasal, transformando la encuesta original de 20 a 22 preguntas $^{4,5}$.

El uso de "subescalas" de la encuesta SNOT, es decir, la evaluación de características clínicas a

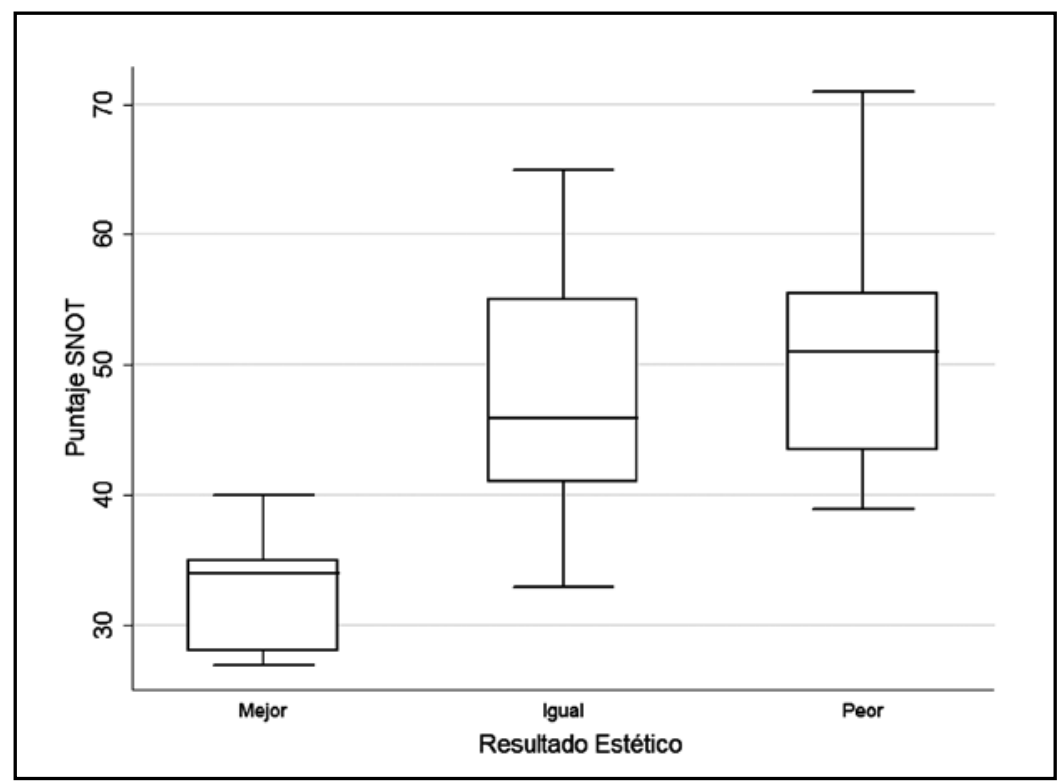

Figura 2. Relación entre la percepción estética y funcional en los pacientes sometidos a reducción de una fractura nasal. 
partir de determinadas preguntas incluidas en la encuesta, ha sido evaluado con anterioridad por Browne y cols ${ }^{6}$, quienes a partir de un estudio prospectivo de 87 pacientes con rinosinusitis en los cuales se utilizó ciertas subescalas, se apreció buena correlación entre los aspectos de calidad de vida con estos puntos analizados mediante la encuesta. En el caso del SNOT-22, utilizamos para este estudio aquellas preguntas que aluden a la presencia de obstrucción nasal (pregunta 4 y 5) y aquellas enfocadas hacia la existencia de roncopatía (preguntas 14 a 20). Este último punto, resultó poco relevante pues muchos de los que refirieron roncopatía la presentaban con anterioridad a la fractura.

No existen aplicaciones de esta escala en fracturas nasales. Hung y cols ${ }^{7}$, evaluaron el grado de satisfacción de los pacientes con la reducción de una fractura nasal mediante encuesta cualitativa sobre su autopercepción estética, encontrando que por lo menos un tercio de ellos presentaban insatisfacción por el resultado, de los cuales casi la totalidad consideraría una rinoseptoplastía para mejorar el resultado.

Los resultados estéticos y funcionales de las reducciones cerradas de las fracturas nasales son en general limitados, aunque reconocemos que estéticamente los cambios son mínimos, lo cual no siempre es compartido por nuestros pacientes. En nuestra serie, el $47,62 \%$ de los pacientes consideró su resultado como peor que antes. Según Fernandes ${ }^{8}$, la insatisfacción de los pacientes con reducciones cerradas de fractura nasal alcanza el $62 \%$, mientras que en aquellos en los que se realiza una rinoseptoplastía inmediata para reducir la fractura, la insatisfacción sólo alcanza el $11,5 \%$. Por estos motivos, se debería incorporar fuertemente el uso de rinoseptoplastía como manejo inicial de las fracturas nasales, para mejorar los resultados funcionales y estéticos de estos pacientes.

En general los estudios sobre fracturas nasales se enfocan en los aspectos estéticos, olvidando que la nariz también juega un rol importante en la vía aérea. Chun y cols ${ }^{9}$, correlacionaron las fracturas nasales con la funcionalidad posterior a la reducción, en términos de obstrucción nasal según el tipo de fractura. Ellos encontraron que a mayor compromiso óseo y desviación septal mayor obstrucción nasal posterior a la reducción se encuentra.

Nuestros resultados clínico-epidemiológicos concuerdan con la descripción clásica, en cuanto a la predominancia en el sexo masculino, su relación con los mecanismos de producción de la fractura nasal y su manejo general ${ }^{1,2,3,10}$.

No comparamos la técnica de reducción (abierta versus cerrada) ya que tuvimos sólo 2 casos que recibieron una reducción abierta de su fractura nasal. Tampoco pudimos comparar el tipo de anestesia utilizada, por los mismos motivos. Evidentemente se necesitarían estudios enfocados a comparar los resultados de estas técnicas (quirúrgica y anestésica) para fundamentar aún más la decisión por una u otra, según el caso lo amerite.

\section{CONCLUSIÓN}

En términos generales consideramos el resultado estético y funcional como no satisfactorio. En nuestro trabajo es posible apreciar que los resultados del puntaje SNOT se correlaciona con la autopercepción estética y funcional (obstrucción nasal) del paciente, posicionando esta escala de evaluación como una importante herramienta en el análisis del resultado de la reducción de una fractura nasal.

\section{BIBLIOGRAFÍA}

1. LeE KJ, ed. Essential Otolaryngology, Head and Neck Surgery. $9^{\text {th }}$ ed. Mc Graw Hill, 2008; 404.

2. Belleza WG, Kalman S. Otolaryngologic emergencies in the outpatient setting. Med Clin North Am 2006; 90(2): 329-53.

3. Kucik CJ, Clenney T, Phelan J. Management of acute nasal fractures. Am Fam Physician 2004; 70(7): 1315-20.

4. Buckland JR, Thomas S, Harries PG. Can the Sino-nasal Outcome Test (SNOT-22) be used as a reliable outcome measure for successful septal surgery? Clin Otolaryngol Allied Sci 2003; 28(1): 43-7. 
5. Browne JP, Hopkins C, Slack R, et al. Healthrelated quality of life after simple nasal polypectomy or nasal polypectomy with additional sino-nasal surgery: a prospective cohort study with three year follow up. Laryngoscope 2006; 297-302.

6. Browne JP, Hopkins C, Slack R, Cano SJ. The Sino-Nasal Outcome Test (SNOT): can we make it more clinically meaningful? Otolaryngol Head Neck Surg 2007; 136(5): 736-41.

7. Hung $T$, Chang $W$, Vlantis AC, Tong MC, van Hasselt CA. Patient satisfaction after closed reduction of nasal fractures. Arch Facial Plast Surg 2007; 9(1): 40-3.

8. Fernandes SV. Nasal fractures: the taming of the shrewd. Laryngoscope 2004; 114(3): 587-92.

9. Chun KW, Han SK, KIM SB, KIM WK. Influence of nasal bone fracture and its reduction on the airway. Ann Plast Surg 2009; 63(1): 63-6.

10. Mondin V, Rinaldo A, Ferlito A. Management of nasal bone fractures. Am J Otolaryngol 2005; 26: 181-5. 